\title{
PRODUCTS OF PERFECTLY MEAGER SETS AND LUSIN'S FUNCTION
}

\author{
JANUSZ PAWLIKOWSKI
}

(Communicated by Andreas Blass)

\begin{abstract}
In [R] Reclaw solved an old problem of Marczewski by constructing two perfectly meager sets with a non-perfectly meager cartesian product. We show another method for getting such sets.
\end{abstract}

A subset $S$ of a polish (i.e., complete separable metric) space $X$ is perfectly meager if every dense in itself subset of $S$ is meager in itself or, equivalently, if $S$ is meager in each perfect subset of $X$ ([K, p. 516; Mi, p. 213]). An uncountable subset of a polish space is a Lusin set if it has countable intersections with each meager set ([K, p. 525; Mi, p. 204]).

In 1935 Marczewski [Ma] (see also [Mi, p. 222]) asked whether a cartesian product of perfectly meager sets is perfectly meager. Recently Reclaw [R] showed that if a Lusin set exists, then the answer is negative. He constructed a closed subset $D$ of $2^{\omega} \times 2^{\omega}$ such that both projections send Lusin subsets of $D$ to perfectly meager sets. It follows that the projections of a Lusin subset of $D$ provide an answer to Marczewski's question: they are perfectly meager and their product, containing a Lusin subset of $D$, is non-meager in $D$.

Sending Lusin sets to perfectly meager sets recalls a function constructed in 1933 by Lusin [L]. This is a continuous one-to-one function $\Phi$ from the space $\mathcal{N}$ of irrationals into itself which maps Lusin sets of $\mathcal{N}$ to perfectly meager sets. The function $\Phi$ proved to be very useful in generating counterexamples in the theory of Baire subsets of the reals. Sierpinski gives a number of them in his book [S, pp. 63-75]. In the present paper we add one more counterexample. Namely we show that a permutation of coordinates in the cartesian square $\Phi \times \Phi$ yields a negative answer to Marczewski's question.

Theorem. The set $D=\left\{\left(\left(x_{0}, \Phi\left(x_{1}\right)\right),\left(x_{1}, \Phi\left(x_{0}\right)\right)\right): x_{0}, x_{1} \in \mathscr{N}\right\}$ is a closed, homeomorphic to $\mathscr{N}$, subset of $(\mathscr{N} \times \mathscr{N}) \times(\mathscr{N} \times \mathscr{N})$ such that both projections onto $\mathscr{N} \times \mathscr{N}$ send Lusin subsets of $D$ to perfectly meager sets.

Received by the editors September 9, 1988 and, in revised form, February 17, 1989.

1980 Mathematics Subject Classification (1985 Revision). Primary 04A15, 54H05.

Key words and phrases. Perfectly meager, nowheredense, Lusin set. 
Note that our set $D$ is the graph of a Borel automorphism of $\mathscr{N} \times \Phi[\mathcal{N}]$, in particular for each $(x, y) \in \mathscr{N} \times \mathscr{N}$ the sections $D \cap\left(\{(x, y)\} \times \mathscr{N}^{2}\right)$ and $\left(\mathscr{N}^{2} \times\{(x, y)\}\right) \cap D$ consist of at most one point. It follows that the cartesian product of the projections of a Lusin subset of $D$ does not have the Baire property in the restricted sense (see [S, p. 71, Proposition C20] or [K, p. 530, Theorem 3] for a similar reasoning).

The theorem will be proved in a slightly more abstract form in $\S 2$. In $\S 3$ we prove a version of the theorem independent of the existence of Lusin sets. We show (Proposition 3.3) a Borel automorphism of a polish space $X$ such that its graph $D$ is a closed subset of $X \times X$ with the property that each $E \subseteq X$ contains a subset $G$ comeager in $E$ with $(G \times X) \cup(X \times G)$ nowheredense in $D$. (Reclaw's set $D$ also has this property, but it is not the graph of a function.)

We close the paper by showing that Lusin's function can be viewed as the identity on $\mathbf{Q}^{\omega}$, the countable cartesian product of rationals, so our $D$ is in fact the diagonal in $\left(\mathbf{Q}^{\omega}\right)^{2} \times\left(\mathbf{Q}^{\omega}\right)^{2}$.

We start with an easy but useful general observation. We say that a family $\mathscr{F}$ of continuous functions from polish spaces to polish spaces has the lifting property if for any function $f$ from $\mathscr{F}$ and for any polish space $Z$ we have

(i) for any homeomorphism $g$ from $Z$ onto $\operatorname{dom}(f)$ the composition $f \circ g$ is in $\mathscr{F}$,

(ii) the lifting $\bar{f}$ of $f$ to $Z$ defined by $\bar{f}(z, x)=(z, f(x)), z \in Z, x \in$ $\operatorname{dom}(f)$, is in $\mathscr{F}$.

Proposition 2.1. Let the functions $f_{i}: X_{i} \rightarrow Y_{i}, i=0,1$ belong to a family $\mathscr{F}$ which has the lifting property. Then the set

$$
D=\left\{\left(\left(x_{0}, f_{1}\left(x_{1}\right)\right),\left(x_{1}, f_{0}\left(x_{0}\right)\right)\right): x_{0} \in X_{0}, x_{1} \in X_{1}\right\}
$$

is closed, homeomorphic to $X_{0} \times X_{1}$ such that the projections onto $X_{0} \times Y_{1}$ and $X_{1} \times Y_{0}$ belong to $\mathscr{F}$.

Proof. The mapping $F_{i}\left(x_{i}, x_{1-i}\right)=\left(\left(x_{0}, f_{1}\left(x_{1}\right)\right),\left(x_{1}, f_{0}\left(x_{0}\right)\right)\right)$ is a homeomorphism between $X_{i} \times X_{1-i}$ and $D$, and its composition with of the projection onto $X_{i} \times Y_{1-i}$ is the lifting of $f_{1-i}$ to $X_{i}$.

We call a continuous function $f$ from a polish space to a polish space an $L$ function if it maps Lusin subsets of its domain to perfectly meager sets. Lusin's function $\Phi$ is an example of a one-to-one $L$ function.

Proposition 2.2. The family of one-to-one $L$ functions with dense in itself domains has the lifting property.

Proof. Let $f: X \rightarrow Y$ be a one-to-one $L$ function, $X$ without isolated points. Fix a polish space $Z$ and let $S$ be a Lusin subset of $Z \times X$. We shall prove that the lifting $\bar{f}$ of $f$ to $Z$ maps $S$ to a perfectly meager set. 
First note that the projection $\Pi$ along $Z$ is a continuous open map such that the inverse images of points are meager. Therefore $\Pi$ sends Lusin sets to Lusin sets. So $\Pi[S]$ is a Lusin subset of $X$ and hence the set $f[\Pi[S]]$ must be perfectly meager.

Now let $A$ be a fixed dense in itself subset of $\bar{f}[S]$. We want to show that $A$ is meager in itself. Each horizontal section of $A$ is countable because it is contained in $\bar{f}[S \cap(Z \times\{x\})]$ for some $x \in X$ and $S \cap(Z \times\{x\})$ is countable as the intersection of the Lusin set $S$ with a meager subset of $Z \times X$. So if $\Pi[A]$ is countable, then $A$ itself is countable, and being dense in itself must be meager in itself.

If $\Pi[A]$ is uncountable, we decompose it into two sets: $A_{0}$-dense in itself and $A_{1}$-countable. $A_{0}$ being a dense in itself subset of the perfectly meager set $f[\Pi[S]]$ is meager in itself and hence meager in $\Pi[A]$. Therefore $A \cap\left(Z \times A_{0}\right)$ is meager in $A$ as the inverse image by an open continuous map (namely $\Pi \mid A$ ) of a meager subset of $\Pi[A]$.

The set $A \cap\left(Z \times A_{1}\right)$ is also meager in $A$ because it is a countable subset of a dense in itself set $A$ (recall that $A_{1}$ and all horizontal sections of $A$ are countable.)

So $A$ is composed of two meager in $A$ subsets and therefore $A$ is meager in itself.

Note that the family of all $L$ functions does not have the lifting property. A contraction of a space to one point provides a counterexample. However we can replace "one-to-one" restriction by "inverse images of points are meager." In the proof we only have to change the point $\{x\}$ to a meager subset of $X$.

Proof of theorem. The theorem follows by Propositions 2.1 and 2.2 and the fact that Lusin's function $\Phi$ is a one-to-one $L$ function.

Lusin's function $\Phi$ has a property which makes it interesting also in the absence of Lusin sets: each subset $E$ of the counterdomain contains a subset $G$ comeager in $E$ such that $\Phi^{-1}[G]$ is nowheredense ([Mi, p. 226]). Continuous functions from polish spaces to polish spaces with this property will be called thin. Thin functions are $L$-functions ([Mi, p. 226]).

Proposition 3.1. The family of thin functions has the lifting property.

The proposition follows easily from the lemma given below.

Lemma. A function $f: X \rightarrow Y$ is thin iff each basic open set $U \subseteq X$ contains an open subset $V$ such that $f[V]$ is meager in $f[U]$.

Proof. Only the if direction needs proving. Fix $E \subseteq Y$. Let

$$
H=\bigcup\{V: V \text { open in } X \text { and } f[V] \text { is meager in } E\} .
$$


It is enough to show that $H$ is open dense in $X$. To get this, let $U$ be a basic open subset of $X$. If $f[U] \subseteq \bar{E}$, let $V \subseteq U$ be any basic open set such that $f[V]$ is meager in $f[U]$. Then $f[V]$ is also meager in $E$. If $f[U] \bar{E} \neq \varnothing$, let $V=f^{-1}[Y \backslash \bar{E}] \cap U$. Then $V$ is nonempty and $f[V]$, being disjoint with $E$, is meager in $E$. So each basic open set $U \subseteq X$ contains an open subset $V$ such that $f[V]$ is meager in $E$. It follows that $H$ is an open dense set.

Lusin's function has an even stronger property than just being thin. It sends every open set to a set which is meager in itself ([K, p. 439(i)]). We shall call such functions strongly thin. It is clear that the family of strongly thin functions has the lifting property. It is less obvious that not all thin functions are strongly thin.

Proposition 3.2. Let $Y$ be a polish space which is dense in itself. There exist a polish space $X$ and a one-to-one thin function from $X$ onto $Y$.

Proof. We can find in $Y$ a system $\left\{P_{\sigma}: \sigma \in \omega^{<\omega}\right\}$ of perfect sets such that $P_{\varnothing}=Y, \sigma \subseteq \tau \rightarrow P_{\tau} \subseteq P_{\sigma}$, each $P_{\tau-i}$ is nowheredense in $P_{\tau}$ and $\bigcup_{i} P_{\tau \frown i}$ is dense in $P_{\tau}([\mathrm{S}, \mathrm{p} .65]$; [ $\left.\mathrm{Mi}, \mathrm{p} .226]\right)$. Let $X$ be a polish space which is obtained from $Y$ by declaring that all $P_{\tau}$ 's are open. (Given a countable family $\mathscr{A}$ of Borel sets in a polish space, we can always enrich the topology to a new polish topology in which sets from $\mathscr{A}$ are open; see [K, p. 448, the proof of Theorem 1]). We claim that the identity function from $X$ to $Y$ is thin. To see this note that any nonempty $X$-open set $U$ must contain a nonempty set $W \cap P_{\tau}$ for some $Y$-open set $W$. Let $i$ be such that the set $V=W \cap P_{\tau \frown i}$ is nonempty. We have $V \subseteq W \cap P_{\tau} \subseteq U$. Moreover $V$ is an $X$-open set which is $Y$-meager in $W \cap P_{\tau}$. So it is $Y$-meager in $U$.

Clearly such a function as above cannot be strongly thin because its range is not meager in itself.

By combining Propositions 2.1, 3.1 and 3.2 we get the following:

Proposition 3.3. There exist a polish space $X$ and a Borel automorphism of $X$ such that its graph $D$ is a closed subset of $X \times X$ with the property that the projections restricted to $D$ are thin functions.

Following a remark of a student of Cichon that the range of Lusin's function is homeomorphic to $\mathbf{Q}^{\omega}$, we show that Lusin's function, when viewed properly, is the identity on the set $\mathbf{Q}^{\omega}$. This gives rise to a particularly simple proof of the basic property of Lusin's function.

Let $\mathscr{M}$ be $\mathbf{Q}^{\omega}, \mathbf{Q}$ bearing its standard topology. As $\mathscr{N}$, the Baire space of irrationals, we also take $\mathbf{Q}^{\omega}$, but equip $\mathbf{Q}$ with the discrete topology.

Proposition 4.1.

(a) $\mathscr{N}$-open sets are $\mathscr{M}$-meager in themselves. 
(b) Any set $E \subseteq \mathbf{Q}^{\omega}$ contains a set $G \subseteq E$ which is $\mathscr{M}$-comeager in $E$ and $\mathscr{N}$-nowheredense in $\mathbf{Q}^{\omega}$.

(c) Any $\mathscr{N}$-Lusin subset of $\mathbf{Q}^{\omega}$ is $\mathscr{M}$-perfectly meager.

Proof. It is enough to show (a) for basic $\mathscr{N}$-open sets. Let $U=\left\{t \in Q^{\omega}: \tau \subseteq\right.$ $t\}$, where $\tau$ is a finite sequence of rationals of length $n$. Let $\mathbf{Q}$ have its standard topology. Then $\mathbf{Q}$ is meager in itself. Since the product of a meager in itself space with any space is meager in itself, $U=\{\tau\} \times \mathbf{Q} \times \mathbf{Q}^{\omega \backslash(n+1)}$ is meager in itself in the $\mathscr{M}$ topology.

Note that by this proposition the identity on $\mathbf{Q}^{\omega}$ is strongly thin when its domain and range have $\mathscr{N}$ and $\mathscr{M}$ topologies respectively. Also, when we topologize $\left(\mathbf{Q}^{\omega}\right)^{2} \times\left(\mathbf{Q}^{\omega}\right)^{2}$ in the manner $(\mathscr{N} \times \mathscr{M}) \times(\mathscr{M} \times \mathscr{N})$, then the diagonal

$$
D=\left\{((x, y),(x, y)): x, y \in \mathbf{Q}^{\omega}\right\}
$$

has the property that the projections act on $D$ as strongly thin functions, i.e. send open subsets of $D$ to sets meager in themselves.

\section{ACKNOWLEDGMENTS}

I am grateful to Jacek Cichon and Michał Morayne for a very stimulating discussion and to Ireneusz Reclaw for patiently explaining to me his result. I am also indebted to Jan Mycielski and Andreas Blass for their influence on the final version of this paper.

\section{REFERENCES}

[K] K. Kuratowski, Topology, vol. 1, Academic Press, 1966.

[L] N. Lusin, Sur les ensembles toujours de première catégorie, Fund. Math. 21 (1933), 114-126.

[Ma] E. Marczewski (Szpilrajn), Problem 68, Fund. Math. 24 (1935), 579.

[Mi] A. W. Miller, Special subsets of the real line, in Handbook of Set-Theoretic Topology, K. Kunen and J. E. Vaughan, eds., North-Holland, 1984, 201-233.

[R] I. Reclaw, Products of perfectly meager sets, preprint, 1987.

[S] W. Sierpiński, Hypothèse du continu, Chelsea, 1956.

Instytut Matematyczny, Universytetu Wroclawskiego, Pl. Grunwaldzki 2/4, 50-384 Wroclaw, Poland 80309

Current address: Department of Mathematics, University of Colorado, Boulder, Colorado 